09,03

\title{
Расчеты из первых принципов и экспериментальное исследование методом спектральной эллипсометрии электронных свойств монокристаллов $\mathrm{CdGa}_{2} \mathrm{~S}_{4}$
}

\author{
(C) З.А. Джахангирли ${ }^{1,2}$, И.А. Мамедова ${ }^{1}$, Ш.Т. Гусейнова ${ }^{1}$, Т.Г. Керимова ${ }^{1}$, Р.Г. Сеидов ${ }^{1}$, \\ Н.Т. Мамедов ${ }^{1}$, Н.А. Абдуллаев ${ }^{1,2, \text { ฯ }}$ \\ ${ }^{1}$ Институт фоизики НАН Азербайджана, \\ Баку, Азербайджан \\ ${ }^{2}$ Бакинский государственный университет, \\ Баку, Азербайджан \\ ๑E-mail: abnadir@mail.ru
}

Поступила в Редакцию 23 сентября 2021 г.

В окончательной редакции 23 сентября 2021 г.

Принята к публикации 6 ноября 2021 г.

\begin{abstract}
Электронные свойства монокристаллов $\mathrm{CdGa}_{2} \mathrm{~S}_{4}$ исследованы экспериментально с использованием спектральной эллипсометрии, а также теоретически из первых принципов с использованием теории функционала плотности (DFT). С помощью эллипсометрических исследований в интервале энергий $0.7-6.5 \mathrm{eV}$ определены мнимые и действительные части диэлектрической функции вдоль и перпендикулярно оптической оси, дисперсия коэффициентов преломления, экстинкции и поглощения. Оценена ширина прямой запрещенной зоны. Зонная структура, происхождение энергетических состояний, оптические функции и проецированные на атомы парциальные плотности состояний (PDoS) определены расчетами из первых принципов. Теоретически рассчитанные результаты сравнены с экспериментальными данными настоящей работы, полученными методом спектральной эллипсометрии.
\end{abstract}

Ключевые слова: спектральная эллипсометрия, эллипсометрические углы, диэлектрическая функция, коэффициент преломления, коэффициент экстинкции, зона Бриллюэна, зонная структура.

DOI: 10.21883/FTT.2022.03.52096.211

\section{1. Введение}

Монокристаллы $\mathrm{CdGa}_{2} \mathrm{~S}_{4}$ относятся к группе соединений $A^{\mathrm{II}} B_{2}^{\mathrm{III}} C_{4}^{\mathrm{VI}}(A-\mathrm{Zn}, \mathrm{Cd} ; B-\mathrm{In}, \mathrm{Ga} ; C-$ $\mathrm{S}, \mathrm{Se}, \mathrm{Te})$, привлекающих внимание исследователей в связи с возможностью их использования в полупроводниковом приборостроении [1]. Для этих соединений характерны двойное лучепреломление, значительные величины коэффициента нелинейной восприимчивости и яркая фотолюминесценция [2]. Благодаря большой ширине запрещенной зоны $(2-4.0 \mathrm{eV})$ и высокой фоточувствительности, на их основе созданы детекторы ультрафиолетового излучения, использующиеся в медицине, биологии, космической физике и других областях. Кристаллы группы $A^{\mathrm{II}} B_{2}^{\mathrm{III}} C_{4}^{\mathrm{VI}}$, и $\mathrm{CdGa}_{2} \mathrm{~S}_{4}$ в частности, являются перспективными материалами для создания перестраиваемых узкополосных светофильтров благодаря наличию т.н. „изотропной точки“, в которой пересекаются кривые дисперсии обыкновенного и необыкновенного показателей преломления [3]. В последнее время кристаллы $\mathrm{CdGa}_{2} \mathrm{~S}_{4}$ и $\mathrm{CdGa}_{2} \mathrm{Se}_{4}$ позиционируются как материалы для солнечных батарей [4].

Ширина запрещенной зоны $\mathrm{CdGa}_{2} \mathrm{~S}_{4}$ экспериментально изучена в работах [5-7]. В [5] ширина запрещенной зоны $E_{g}$ монокристаллов $\mathrm{CdGa}_{2} \mathrm{~S}_{4}$, определенная из исследований спектров поглощения, оказалась равной $3.5 \mathrm{eV}$ при $300 \mathrm{~K}$, а в [6,7] ширина запрещенной зоны $\mathrm{CdGa}_{2} \mathrm{~S}_{4}$, определенная также из исследований спектров поглощения при $300 \mathrm{~K}$, определена как 3.43 и $3.15 \mathrm{eV}$ соответственно.

Впервые ширина запрещенной зоны была теоретически рассчитана псевдопотенциальным методом [8], и для прямой запрещенной зоны было получено значение $3.43 \mathrm{eV}$. Дальнейшие теоретические расчеты электронной зонной структуры производились из первых принципов. В работе [9] приводятся данные вычислений в приближении локальной плотности (local-density approximation — LDA), и получено корректированное значение ширины прямой запрещенной зоны в точке $\Gamma-3.3 \mathrm{eV}$. В работах $[10,11]$ на основе расчетов $a b$ initio электронных и оптических свойств $\mathrm{CdGa}_{2} \mathrm{~S}_{4}$ методом DFT (Density Functional Theory) приводятся значения ширин прямой запрещенной зоны в точке $\Gamma-2.118$ и $2.2 \mathrm{eV}$, соответственно. Рассчитанные действительные части статических диэлектрических постоянных в работе $[10] \varepsilon_{1}(0)_{\perp}=4.292, \varepsilon_{1}(0)_{\|}=4.229$, а коэффициентов преломления $n(0)_{\perp}=2.072$ и $n(0)_{\|}=2.056$. В [11] представлены несколько отличные величины рассчитанных действительных частей статических диэлектрических постоянных: $\varepsilon_{1}(0)_{\perp}=7.32, \varepsilon_{1}(0)_{\|}=5.35$, а коэффициентов преломления $n(0)_{\perp}=2.70$ и $n(0)_{\|}=2.31$. 
Таким образом, несмотря на большой объем экспериментальных данных по кристаллам $\mathrm{CdGa}_{2} \mathrm{~S}_{4}$, в литературе имеется разброс в значениях основных оптических параметров (ширины запрещенной зоны, диэлектрических постоянных, коэффициентов преломления и т.д.), что требует дополнительных исследований. В данной статье представлены результаты экспериментального и теоретического исследования электронной зонной структуры и оптических свойств монокристаллов $\mathrm{CdGa}_{2} \mathrm{~S}_{4}$. Для экспериментальных исследований был использован метод спектральной эллипсометрии, являющийся одним из точных методов определения оптических характеристик кристаллов.

\section{2. Эксперимент}

Образцы для измерений были синтезированы из исходных компонентов $\mathrm{Cd}$, $\mathrm{Ga}$ и $\mathrm{S}$, взятых в стехиометрическом соотношении в графитизированных кварцевых ампулах. Выращивание монокристаллов производилось методом газотранспортных реакций, в качестве транспортера использовался кристаллический йод.

Рентгендифрактометрические исследования проводились на приборе Bruker D8 Advance. $\mathrm{CdGa}_{2} \mathrm{~S}_{4}$ кристаллизуется в тетрагональной структуре с пространственной группой симметрии $\bar{I} 4$. Образцы имели форму прямоугольного параллелепипеда с размерами примерно $8 \times 3 \times 3 \mathrm{~mm}$. Тетрагональная ось была направлена вдоль наибольшего размера. Параметры элементарной ячейки, определенные из данных рентгеновской дифракции [12], и оптимизированные равновесные значения этих параметров из теоретических расчетов приведены в табл. 1.

Измерения спектров комбинационного рассеяния в $\mathrm{CdGa}_{2} \mathrm{~S}_{4}$ были проведены на конфокальном рамановском микроспектрометре Nanofinder 30 (Tokyo Instr., Japan). В качестве источника возбуждения использовался лазер $\mathrm{Nd}$ :YAG с выходной длиной волны излучения на второй гармонике $\lambda=532 \mathrm{~nm}$ и максимальной мощностью $10 \mathrm{~mW}$. Спектральное разрешение при использовании дифракционной решетки 1800 lines $/ \mathrm{mm}$ было не хуже $0.5 \mathrm{~cm}^{-1}$. Детектором рассеянного излучения являлась охлаждаемая (термоэлектрическим способом до $\left.-100^{\circ} \mathrm{C}\right) \mathrm{CCD}$-камера $(1024 \times 128$ pixels $)$, работающая в режиме счета фотонов. Измерения спектров комбина-

Таблица 1. Структурные параметры $\mathrm{CdGa}_{2} \mathrm{~S}_{4}$

\begin{tabular}{l|c|c|c}
\hline \multicolumn{1}{c|}{$\AA, \AA$} & $c, \AA$ & $c / a$ & Лит. \\
\hline 5.56 & 10.00 & 1.799 & {$[13]$} \\
5.536 & 10.16 & 1.835 & {$[14]$} \\
5.644 & 10.347 & 1.833 & {$[10]$} \\
5.55 & 10.19 & 1.834 & дан. раб. (эксп.) \\
5.4272 & 9.9297 & 1.8296 & дан. раб. (теория)
\end{tabular}

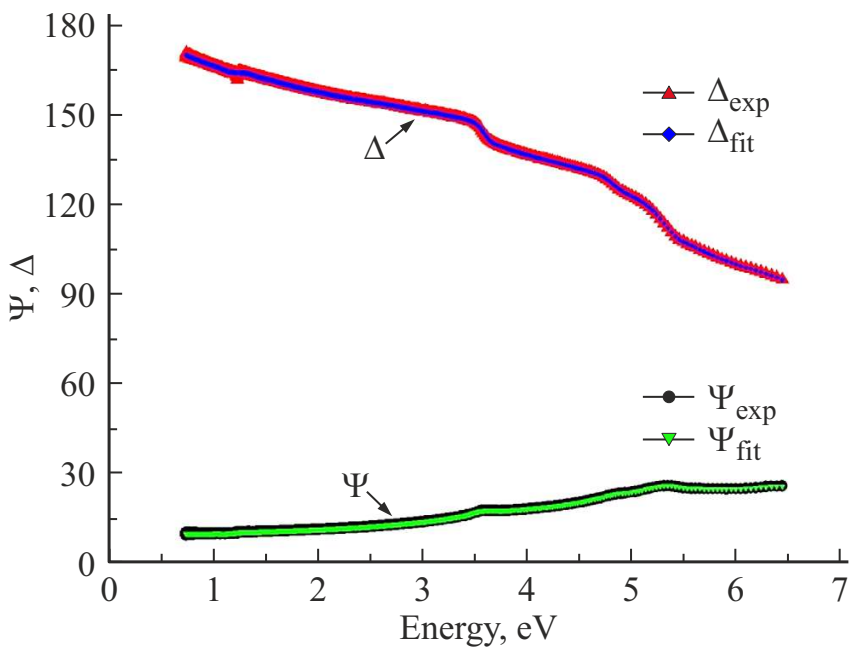

Рис. 1. Экспериментальные и расчетные данные эллипсометрических параметров $\Psi$ и $\Delta$ для $\mathrm{CdGa}_{2} \mathrm{~S}_{4}$ в интервале энергий $0.7-6.5 \mathrm{eV}$.

ционного рассеяния света производились в геометрии обратного рассеяния.

Характеризация образцов $\mathrm{CdGa}_{2} \mathrm{~S}_{4}$ методами рентгеновской дифракции и комбинационного рассеяния света свидетельствует о высоком качестве образцов и подробно описана в предыдущей работе [15].

Для исследования оптических характеристик кристаллов $\mathrm{CdGa}_{2} \mathrm{~S}_{4}$ нами были проведены спектральные эллипсометрические исследования, основанные на определении изменения состояния поляризации света в результате взаимодействия его с поверхностью кристаллов при отражении. Измерения проводились на эллипсометре оптического диапазона M-2000 DI (J.A. Woollam Co, Inc.). Спектральная зависимость эллипсометрических параметров $\Delta$ и $\Psi$ снималась в диапазоне энергий фотонов $0.7-6.5 \mathrm{eV}$ с шагом $50 \mathrm{meV}$ при углах падения излучения в диапазоне $60^{\circ}-75^{\circ}$ с шагом $5^{\circ}$.

Основное уравнение эллипсометрии, связывающее между собой эллипсометрические параметры $\Delta, \Psi$ и комплексные значения коэффициентов отражения Френеля $r_{p}$ и $r_{s}$, для $p$ - и $s$-компонентов эллиптически поляризованного света, записывается в виде $[16,17]$ :

$$
\operatorname{tg}(\Psi) \exp (\mathrm{i} \Delta)=\frac{r_{p}}{r_{s}} .
$$

Экспериментальные и расчетные данные эллипсометрических параметров $\Psi$ и $\Delta$ для $\mathrm{CdGa}_{2} \mathrm{~S}_{4}$ в интервале энергий $0.7-6.5 \mathrm{eV}$ показаны на рис. 1.

Рассчитанные из эллипсометрических измерений величины оптических характеристик (действительная и мнимая части диэлектрической функции, коэффициенты экстинкции и поглощения и показатель преломления) будут даны ниже совместно с данными теоретических расчетов из первых принципов. 


\section{3. Метод расчета}

Расчеты $a b$ initio электронных и оптических свойств проводились на основе DFT [18] с использованием метода полнопотенциальных линеаризованных дополненных плоских волн (FP-LAPW) [19], реализованного в программном коде Wien2k [20]. Обменно-корреляционное взаимодействие описывалось в обобщенном градиентном приближении (GGA) [21]. Радиусы маффин-тин (MT) сфер были выбраны равными 2.5 a.u. для $\mathrm{Cd}, 2$ a.u. для $\mathrm{Ga}$ и S. Разложения потенциала и плотности заряда внутри МТ-сфер производились с $l_{\max }=10$. В разложении базисных функций были использованы $R_{\mathrm{MT}} K_{\max }=7$, который определяет число базисных функций, где $R_{\mathrm{MT}}-$ наименьший из всех радиусов атомной сферы, а $K_{\max }$ - максимальный волновой вектор плоской волны. Для самосогласованных расчетов были использованы $186 k$-точек в неприводимой части зоны Бриллюэна $(\mathrm{BZ})$, что соответствует $1500 k$-точкам в BZ. Самосогласованные расчеты продолжались до тех пор, пока сходимость полной энергии достигалась $10^{-4} \mathrm{Ry}$.

\section{4. Электронная структура и оптические функции}

Зонная структура и проектированные на атомы парциальные плотности состояний (PDoS) показаны на рис. 2 и 3 соответственно. Зонная структура $\mathrm{CdGa}_{2} \mathrm{~S}_{4}$ (рис. 2) обладает рядом характерных особенностей: валентная зона состоит условно из пяти подзон. Самая нижняя подзона, практически это бездисперсионные состояния около $-14 \mathrm{eV}$, обязанные своим происхождением в основном $d$-состояниям атомов $\mathrm{Ga} 1$ и $\mathrm{Ga} 2$, вклад которых не вполне равноценен - вклад $d$-состояний атомов $\mathrm{Ga} 1$ более чем в 3 раза больше, с некоторой долей вклада $s$-состояний атомов халькогена S. Вторая подзона - это группа валентных зон между энергиями -13 и $-11 \mathrm{eV}$, которые происходят из $s$-состояний атомов халькогена $\mathrm{S}$ и $d$-состояний атомов Ga1 и Ga2 (рис. 3 ). Как следует из $\mathrm{PDoS}$ (рис. 3), почти бездисперсионные состояния около $-7 \mathrm{eV}$ обязаны своим происхождением $d$-состояниям атомов $\mathrm{Cd}$. Анализ парциальной плотности состояний показывает, что группа валентных зон в диапазоне энергий примерно от -6.5 до $-4.5 \mathrm{eV}$ происходит из $s$-состояний $\mathrm{Ga} 1$ и $\mathrm{Ga} 2$, а также $p$-состояний атомов халькогена $\mathrm{S}$.

И наконец, самую верхнюю группу валентных зон можно приписать $p$-состояниям атомов халькогена $\mathrm{S}$ c малым вкладом $p$ - и $d$-состояний $\mathrm{Cd}$ и $p$-состояний $\mathrm{Ga} 1$ и $\mathrm{Ga} 2$.

Потолок валентной зоны и абсолютный минимум зоны проводимости находится в точке $Г$ и, соответственно, согласно расчетам $\mathrm{CdGa}_{2} \mathrm{~S}_{4}$ является прямозонным полупроводником с шириной запрещенной зоны $\sim 2.2 \mathrm{eV}$. Это значение близко к рассчитанным ранее из

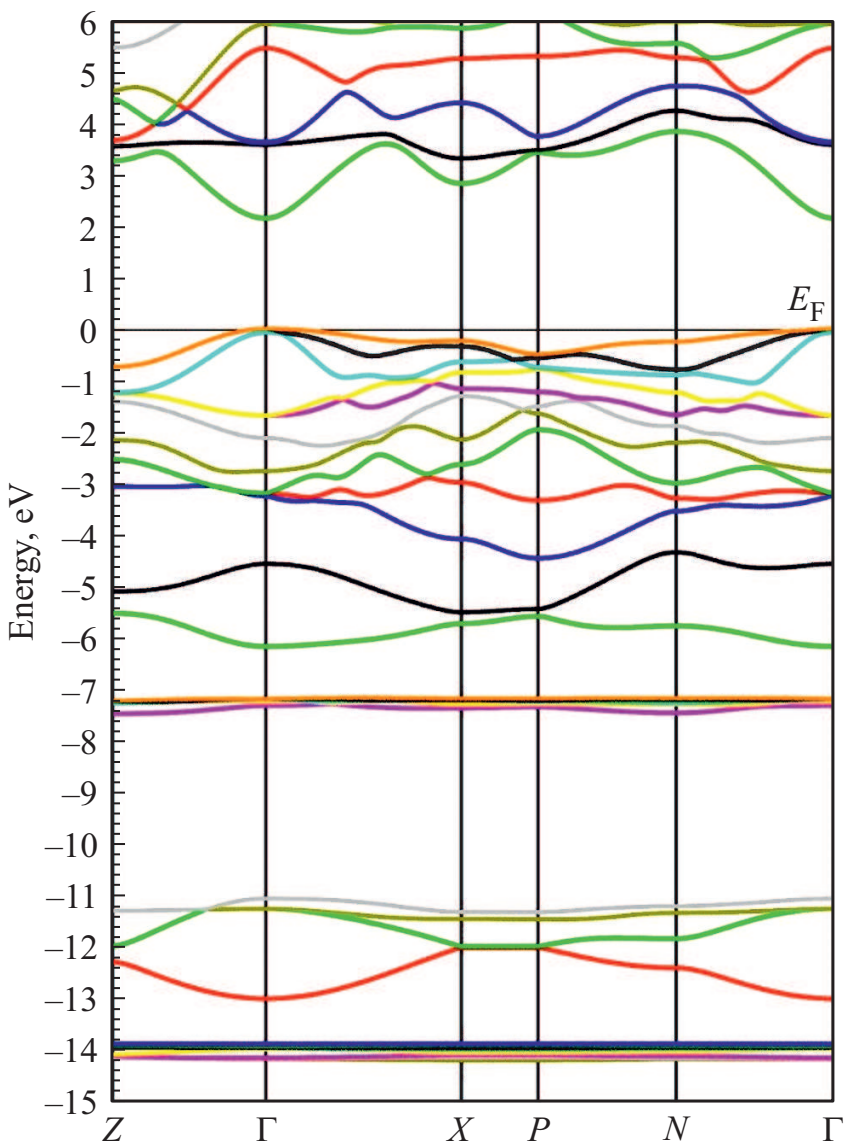

Рис. 2. Зонная структура $\mathrm{CdGa}_{2} \mathrm{~S}_{4}$.

первых принципов значениям $2.118 \mathrm{eV}$ [10] и $2.2 \mathrm{eV}$ [11], но существенно ниже экспериментально определенных $3.3-3.5 \mathrm{eV}$. Общеизвестно, что DFT недооценивает величины ширин запрещенной зоны, в том числе и в этих кристаллах. В работе [9] проводится обзор данных по ширинам запрещенных зон в соединениях дефектных халькопиритов группы $A^{\mathrm{II}} B_{2}^{\mathrm{III}} C_{4}^{\mathrm{VI}}(A-\mathrm{Zn}, \mathrm{Cd}, \mathrm{Hg} ; B-$ $\mathrm{Al}, \mathrm{In}, \mathrm{Ga} ; C-\mathrm{S}, \mathrm{Se}, \mathrm{Te})$ и отмечается существенное несоответствие величин, теоретически вычисленных из первых принципов методом LDA и экспериментально определенных. Отмечается, что причиной является то, что локальный обменно-корреляционный потенциал $v_{\mathrm{xc}}$ заменяется нелокальным оператором собственной энергии $\Sigma_{\mathrm{xc}}$, и эмпирическая поправка на запрещенные зоны вне приближения локальной плотности дает хорошее согласие теоретически вычисленных и экспериментально определенных данных.

Из парциальной плотности состояний также видно, что дно зоны проводимости состоит в основном из $s$-состояний атомов $\mathrm{Ga} 1$ и $\mathrm{Ga} 2$ с некоторой добавкой $p$-состояний атома $\mathrm{S}$.

Сравнение рассчитанной зонной структуры с экспериментальными исследованиями оптических свойств позволяет нам проверить правильность нашей зонной структуры. Собственные значения и собственные век- 


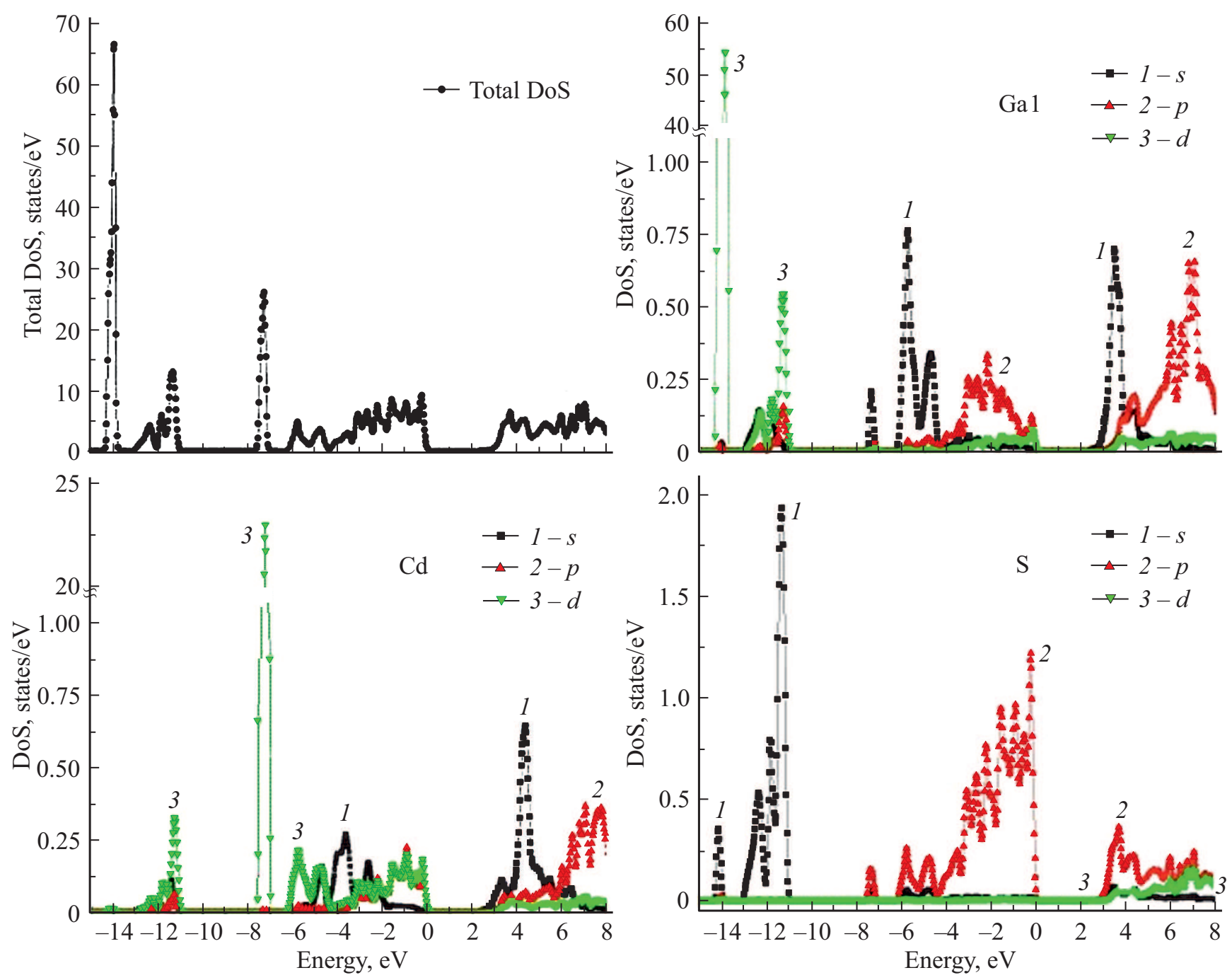

Рис. 3. Полная (DoS) и парциальные плотности состояний (PDoS) атомов $\mathrm{Cd}$, Ga и S. Уровень Ферми соответствует нулевой энергии.

торы, полученные из расчетов зонной структуры, использовались для определения действительной и мнимой частей диэлектрической функции, коэффициентов экстинкции и поглощения и показателя преломления. Рассчитанные из первых принципов и измеренные экспериментально методом спектральной эллипсометрии оптические функции $\mathrm{CdGa}_{2} \mathrm{~S}_{4}$ показаны на рис. 4,5 и 6. Отметим, что только прямые переходы, которые сохраняют импульс, включены в наши расчеты для оптических свойств. Непрямые переходы с участием фононов оказывают очень слабое влияние на край поглощения, так как $\mathrm{CdGa}_{2} \mathrm{~S}_{4}$ является прямозонным полупроводником.

Рассчитанные из первопринципов в спектральной области $0-15 \mathrm{eV}$ и измеренные экспериментально в области энергий фотонов $0.7-6.5 \mathrm{eV}$ действительные и мнимые части диэлектрической функции $\mathrm{CdGa}_{2} \mathrm{~S}_{4}$ для различных поляризаций падающего света (вдоль и перпендикулярно тетрагональной оси $c$ ) показаны на рис. 4.
Основные пики рассчитанной действительной и мнимой части расположены около 3.4 и $5.5 \mathrm{eV}$ соответственно. Используя известные значения действительной $\operatorname{Re} \varepsilon\left(\varepsilon_{\mathrm{r}}\right)$ и мнимой $\operatorname{Im} \varepsilon\left(\varepsilon_{\mathrm{i}}\right)$ частей диэлектрической функции $\varepsilon$ для кристаллов $\mathrm{CdGa}_{2} \mathrm{~S}_{4}$, нами были рассчитаны показатели преломления $n$ и экстинкции $k$, соответственно, с помощью формул (2) и (3):

$$
\begin{gathered}
n=\sqrt{\frac{\varepsilon_{\mathrm{r}}+\sqrt{\varepsilon_{\mathrm{r}}^{2}+\varepsilon_{\mathrm{i}}^{2}}}{2}}, \\
k=\frac{\varepsilon_{\mathrm{i}}}{\sqrt{2\left(\varepsilon_{\mathrm{r}}+\sqrt{\varepsilon_{\mathrm{r}}^{2}+\varepsilon_{\mathrm{i}}^{2}}\right)}},
\end{gathered}
$$

На рис. 5 приведены данные полученных экспериментально (кривые 1) и рассчитанных из первопринципов (кривые 2) величин показателей преломления $n$ (рис. 5, $a$ и $b$ ) и коэффициентов экстинкции $k$ (рис. 5, $c$ и $d$ ) 

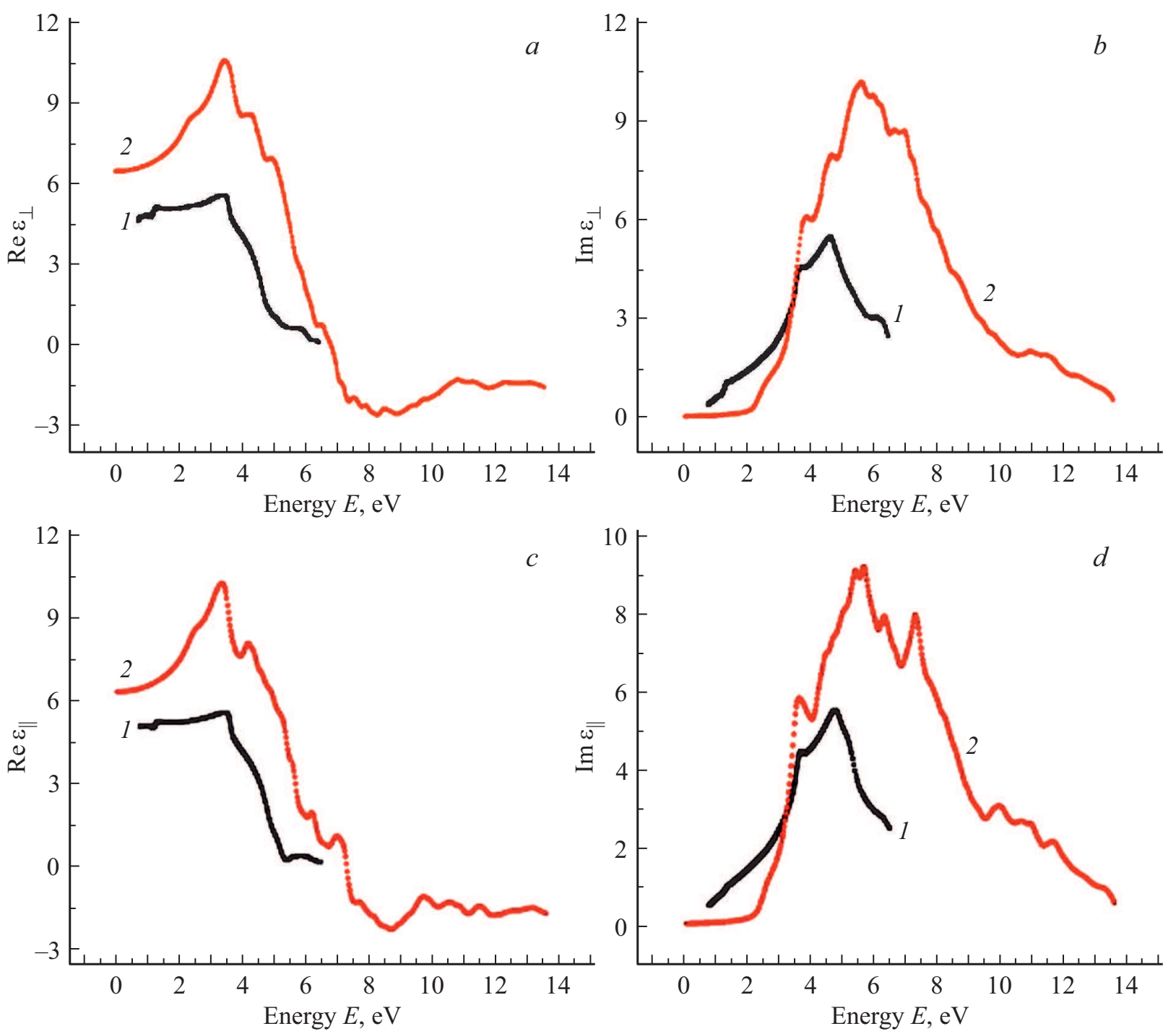

Рис. 4. Реальные и мнимые части диэлектрической функции $\mathrm{CdGa}_{2} \mathrm{~S}_{4}(1-$ эксперимент, 2 - теория; $\|$ - вдоль тетрагональной оси $c, \perp-$ перпендикулярно тетрагональной оси $c)$.

вдоль $(\|)$ и перпендикулярно $(\perp)$ тетрагональной оси $c$ в широкой области энергий.

Отметим, что измеренные показатели преломления в случае $E \perp c$ и $E \| c n_{\perp}$ и $n_{\|}$(кривые 1, рис. 5, $a$ и $b$ ), также как и теоретически вычисленные (кривые 2 , рис. 5, $a$ и $b$ ), показывают одинаковые зависимости $n \perp(E)$ и $n \|(E)$, существенной анизотропии не обнаруживают и достигают максимума около $3.5 \mathrm{eV}$. Теоретические значения статических показателей преломления $n_{\perp}(0)$ и $n_{\|}(0)$ (значение показателя преломления при нулевой энергии) составляют $\sim 2.5$. Измеренные коэффициенты экстинкции в случае $E \perp c$ и $E \| c-k_{\perp}$ и $k_{\|}$(кривые 1$)$, рис. 5, $c$ и $\left.d\right)$, так же как и теоретически вычисленные (кривые 2, рис. 5, $c$ и $d$ ), показывают одинаковые зависимости $k_{\perp}(E)$ и $k_{\|}(E)$ численное совпадение вплоть до энергии $5 \mathrm{eV}$, при этом совпадают положения особенностей на зависимостях $k(E)$ при энергиях 3.6 и $4.8 \mathrm{eV}$. Отметим также, что существенной анизотропии
Таблица 2. Некоторые оптические параметры $\mathrm{CdGa}_{2} \mathrm{~S}_{4}$

\begin{tabular}{l|l|l|l|c}
\hline$\varepsilon_{1}(0)_{\perp}$ & $\varepsilon_{1}(0)_{\|}$ & $n(0)_{\perp}$ & $n(0)_{\|}$ & Лит. \\
\hline 4.292 & 4.229 & 2.072 & 2.056 & {$[10]$} \\
7.32 & 5.35 & 2.70 & 2.31 & {$[11]$} \\
6.473 & 6.307 & 2.544 & 2.511 & дан. раб. (теория)
\end{tabular}

коэффициентов экстинкции $k_{\perp}(E)$ и $k_{\|}(E)$ также не наблюдается. В табл. 2 приведены некоторые оптические параметры $\mathrm{CdGa}_{2} \mathrm{~S}_{4}$.

Для расчета коэффициента поглощения $\alpha$ мы воспользовались соотношением:

$$
\alpha=4 \pi k / \lambda
$$

Здесь $k-$ коэффициент экстинкции, а $\lambda-$ длина волны. 

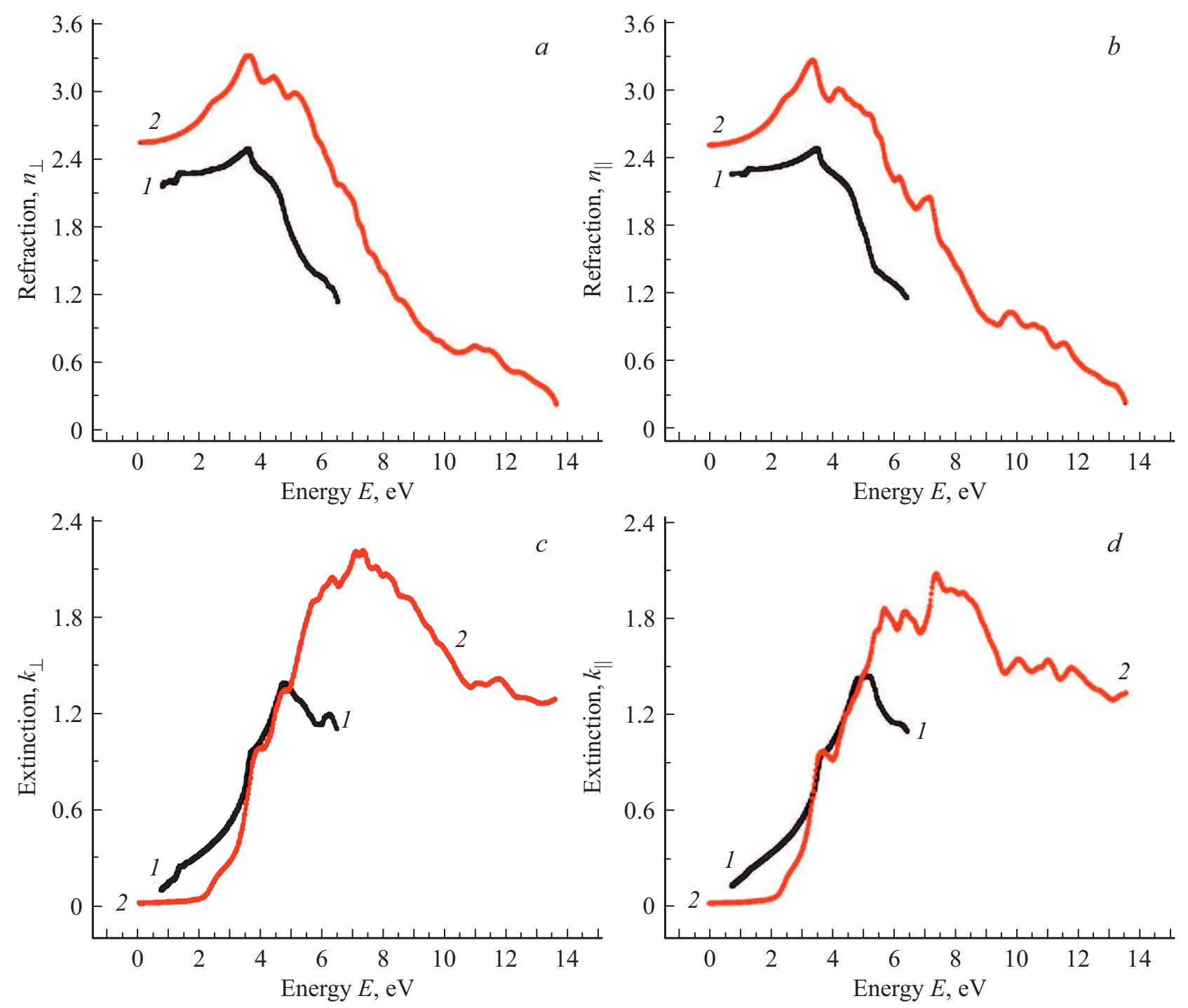

Рис. 5. Показатель преломления $n$ и коэффициент экстинкции $k$ для $\mathrm{CdGa}_{2} \mathrm{~S}_{4}$.

На рис. 6 приведены данные измеренных экспериментально (кривые 1) и рассчитанных из первых принципов (кривые 2) величин коэффициента поглощения $\alpha$ (рис. 6, $a$ и $b$ ), вычисленных по (4).

Высокое значение коэффициента поглощения $\alpha$ $\left(10^{4}-10^{5} \mathrm{~cm}^{-1}\right)$, его резкое увеличение в области энергий $1.5-4 \mathrm{eV}$ свидетельствуют о прямом разрешенном характере запрещенной зоны. Для прямозонного перехода зависимость коэффициента поглощения от энергии фотона выглядит следующим образом:

$$
\alpha \hbar v \sim\left(E_{g}-\hbar v\right)^{1 / 2} .
$$

Экстраполяция из соотношения (5) позволила нам оценить ширины прямой запрещенной зоны монокристаллов $\mathrm{CdGa}_{2} \mathrm{~S}_{4}$ вдоль $(E \| c)$ и перпендикулярно $(E \perp c)$ тетрагональной оси (рис. 6, $c$ и $d$ ). Она оказалась равной соответственно $3.27 \mathrm{eV}$ (для $E \| c$ ) и $3.31 \mathrm{eV}$ (для $E \perp c)$, что согласуется с экспериментальными данными из измерений спектров поглощения [5-7].

\section{5. Заключение}

Нами были проведены экспериментальные исследования оптических свойств монокристаллов $\mathrm{CdGa}_{2} \mathrm{~S}_{4}$ вдоль $(\|)$ и перпендикулярно $(\perp)$ тетрагональной оси $c$ в широкой области энергий $0.7-6.5 \mathrm{eV}$ методом спектральной эллипсометрии, результаты которых были сравнены с расчетами ab initio электронной структуры и оптических функций. По данным эллипсометрических измерений были определены реальная и мнимая части диэлектрической функции, показатель преломления, коээффициенты экстинкции и поглощения. Результаты эксперимента показывают, что $\mathrm{CdGa}_{2} \mathrm{~S}_{4}$ является разрешенным прямозонным полупроводником с шириной запрещенной зоны $\sim 3.3-3.4 \mathrm{eV}$.

Использованием DFT определены электронная зонная структура кристаллов $\mathrm{CdGa}_{2} \mathrm{~S}_{4}$ и проецированные на атомы кадмия, галлия и серы парциальные плотности состояний (PDoS). Показано, что теоретически полу- 

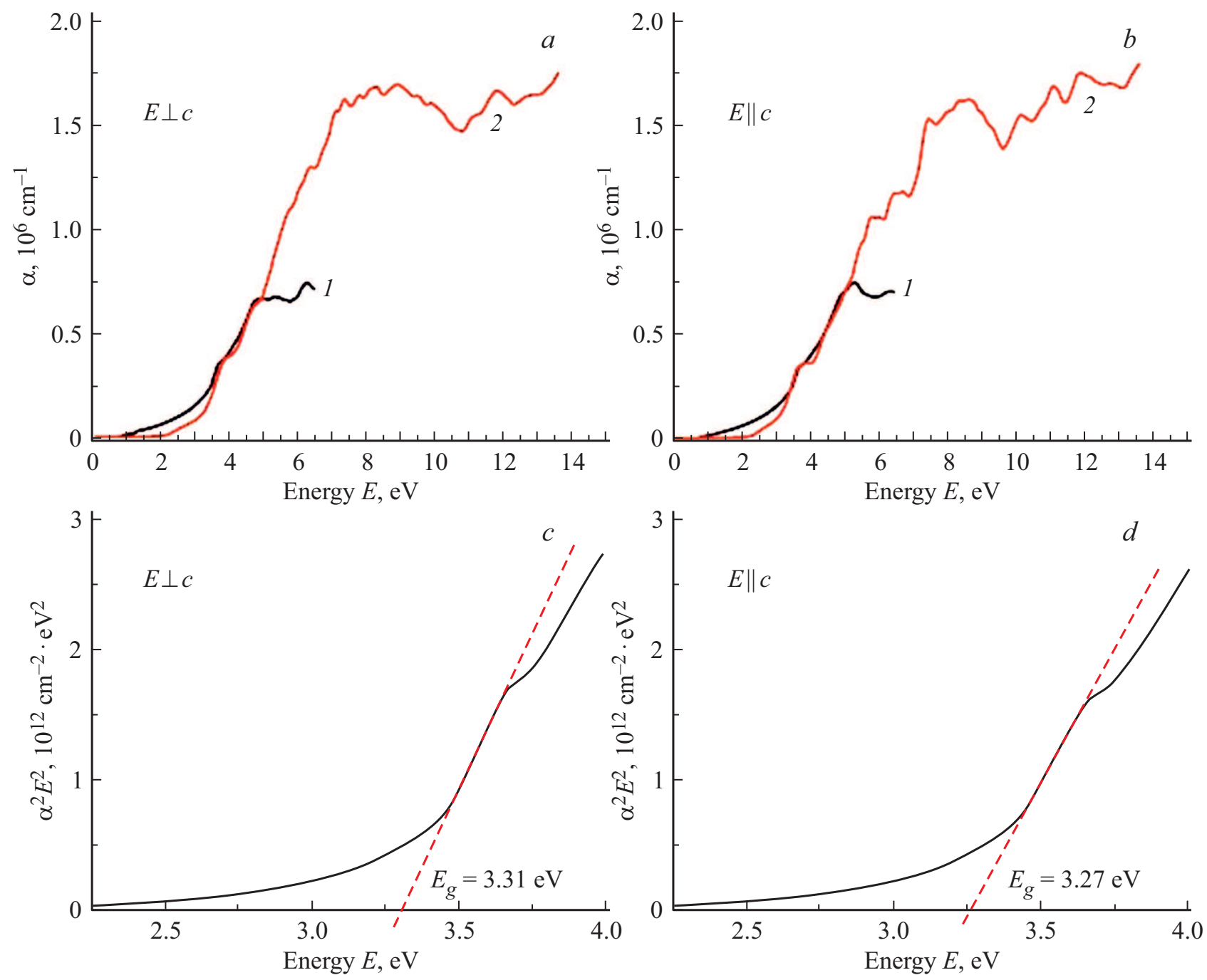

Рис. 6. Коэффициент поглощения $\alpha(a$ и $b)$ и зависимости $\alpha^{2} E^{2}(E)(c$ и $d)$ для $\mathrm{CdGa}_{2} \mathrm{~S}_{4}$.

ченные результаты удовлетворительно согласуются с экспериментально определенными величинами.

\section{Благодарности}

Авторы считают своим долгом выразить благодарность А.С. Бондякову (ОИЯИ, Россия) и Д.А. Кулиеву (Институт физики НАН Азербайджана), а также всему коллективу Дата-центра Института физики НАН Азербайджана, за предоставленные ресурсы и техническую поддержку теоретических расчетов.

\section{Финансирование работы}

Работа выполнена при финансовой поддержке Фонда развития науки при Президенте Азербайджанской республики (грант № EIF-BGM-3-BRFTF-2+/2017-15/02/1).

\section{Конфликт интересов}

Авторы заявляют, что у них нет конфликта интересов.

\section{Список литературы}

[1] А.Н. Георгобиани, С.И. Радауцан, И.М. Тигиняну. ФТП 19, 2, 193 (1985).

[2] H. Park, Y.-S. Kim, S.-C. Hyun, C.-D. Kim, M.-S. Jin, D.-T. Kim, K. Jang, H.-G. Kim, W.-T. Kim. Phys. Status Solid C 3, 8, 2915 (2006).

[3] В.В. Бадиков, И.Н. Матвеев, С.М. Пшеничников, О.В. Рычик, Н.К. Троценко, Н.Д. Устинов. Квантовая электрон. 8, 4, 910 (1981). [Sov. J. Quantum Electronics 11, 4, 548 (1981)].

[4] P. Kumar, J. Sahariya, A. Soni, K.C. Bhamu. Mater. Sci. Forum 900, 69 (2017).

[5] Т.Г. Керимова, Ш.С. Мамедов, И.А. Мамедова. Неорган. материалы 29, 7, 902 (1993).

[6] H. Park, C.-D. Kim. J. Korean Phys. Soc. 45, 2, 427 (2004).

[7] Y.-S. Kim, H. Park, C.-D. Kim. J. Korean Phys. Soc. 41, 3, 349 (2002).

[8] V.L. Panyutin, B.E. Ponedelnikov, A.E. Rozenson, V.I. Chizhikov. Sov. Phys. J. 22, 8, 857 (1979).

[9] X. Jiang, W.R.L. Lambrecht. Phys. Rev. B 69, 3, 035201 (2004). 
[10] S.H. Ma, Z.Y. Jiao, X.Z. Zhang. J. Mater. Sci. 47, 8, 3849 (2012).

[11] S. Mishraa, B. Ganguli. arXiv:1508.00407v1[cond-mat.mtrl-sci], 32 c. (2015).

[12] Г.Г. Гусейнов, Т.Г. Керимова, Р.Х. Нани. Изв. АН Аз. ССР 4, 59 (1980).

[13] H. Hahn, G. Frank, W. Kligler, A.D. Storger. Z. Anorg. Allg. Chem. 279, 241 (1955).

[14] D. Errandonea, R.S. Kumar, F.J. Manjon, V.V. Ursaki, I.M. Tiginyanu. J. Appl. Phys. 104, 6, 063524 (2008).

[15] 3.А. Джахангирли, Т.Г. Керимова, И.А. Мамедова, Н.А. Абдуллаев, Н.Т. Мамедов. ФТТ 60, 11, 2265 (2018).

[16] H. Fujiwara. Spectroscopic Ellipsometry: Principles and Applications. John Wiley and Sons, NJ, USA (2007). Ch. 1.3, p. 5.

[17] А.В. Ржанов, К.К. Свиташев, А.И. Семененко, Л.В. Семененко, В.К. Соколов. Основы эллипсометрии. Наука, Новосибирск (1979). Гл. 1.4. С. 46.

[18] P. Hohenberg, W. Kohn. Phys. Rev. B 136, 3B, 864 (1964).

[19] O.K. Andersen. Phys. Rev. B 12, 8, 3060 (1975).

[20] P. Blaha, K. Schwarz, G.K.H. Madsen, D. Kvasnicka, J. Luitz. WIEN2k, An Augmented Plane Waves + Local Orbitals Program for Calculating Crystal Properties, rev. ed. Vienna University of Technology, Vienna (2008).

[21] J.P. Perdew, A. Zunger. Phys. Rev. B 23, 10, 5048 (1981).

Редактор Е.В. Толстякова 